\title{
Essays
}

\section{The growing dilemma of peer review: a three-generation viewpoint}

\author{
Victor Virlogeux ${ }^{1,2,3,4}$; Christian Trépo ${ }^{1,2,3}$; Pierre Pradat ${ }^{2,3}$ \\ 1. Service d'Hépatologie, Groupement Hospitalier Nord, Hospices Civils de Lyon, Lyon, France \\ 2. Université Claude Bernard Lyon 1, Inserm 1052, CNRS 5286, Centre Léon Bérard, Centre de recherche en cancérologie de \\ Lyon, Lyon, France \\ 3. Centre de Recherche Clinique, Groupement Hospitalier Nord, Hospices Civils de Lyon, Lyon, France \\ 4. Département de Biologie, Ecole Normale Supérieure de Lyon, Lyon, France \\ Correspondence to pierre.pradat@univ-lyon1.fr
}

DOI: 10.20316/ESE.2018.44.17019

\begin{abstract}
The reviewing process is currently facing a major challenge due to a lack of reviewers. Set up to improve the readability and the quality of published manuscripts, peer review has become the gold standard for scientific literature. Historically, this process was introduced by the Royal Society of London in 1662 in order to ensure scientific integrity as modern science needs to be vetted by peers prior to dissemination. Peer review works on a voluntary basis. However, an ever increasing number of manuscripts, the significant time required for each review, conflicts with other workloads, and lack of credit, make finding reviewers more and more difficult. We discuss in this essay several solutions that could help address the current challenges of peer review.
\end{abstract}

\section{Introduction}

On November 11, 2016, more than three months after having submitted a manuscript to a peer-reviewed journal, we received the following message from the editor: "I regret to inform you that after much consideration, your manuscript entitled ... will not be considered for publication. We have sent the paper out for review to over 15 potential reviewers and have been unable to receive a review from any of them. We receive more manuscripts than we can publish and, as a result, we are forced to make priority decisions.... Unfortunately, this situation is becoming frequent since journal editors find it is increasingly difficult to find researchers able and willing to complete timely reviews.

The review process has always been considered as the "gatekeeper" of science. Peer review was introduced by the Royal Society of London in 1662 in the first issue of Philosophical Transactions. ${ }^{1}$ Although the evaluation of scientific literature by invited reviewers has only recently become the gold standard, its origin may be found in these historical practices. ${ }^{2}$ Peer review aims to ensure scientific integrity, vetting scientific results prior to dissemination. However, a recent Nature survey reported that less than $30 \%$ of published, peer-reviewed scientific articles were reproducible. $^{3}$

Peer review works on a voluntary basis. It allows researchers to play a part in the scholarly community and to reciprocate others reviewing their work. ${ }^{4}$ In a survey aiming to identify reasons why people decline to review manuscripts, Tite and Schroter found that the most important factors were conflict with other workload, tight deadline for review completion, and having to review too many manuscripts for other journals. ${ }^{5}$

In another survey of about 2900 researchers, Wiley learned that acceptance to review a manuscript was dependent on the journal impact factor. Disparities were also observed depending on the country, with a large proportion of manuscripts being reviewed by US researchers. The reviewing process was attracting more researchers who were in the early stages of their career because of time concerns and hope for recognition. ${ }^{6}$

For these different reasons, combined with the lack of credit for this time-consuming job, finding reviewers is becoming increasingly difficult, especially for journals with low or moderate impact factors.

\section{How to increase the number of reviewers?}

Several surveys supported by editorial groups have been recently conducted to investigate the issues of peer review and to ask reviewers how this process could be improved. ${ }^{4-7}$

\section{Acknowledgement}

Most of the reviewers believe that peer review is inadequately acknowledged and that the process should carry more weight in the research community. ${ }^{6,7}$ The system of acknowledgement usually consists of publishing the list of contributors online, or sending original certificates and letters of appreciation to top reviewers. Suggestions from the reviewers were to receive more feedback from the journal regarding the quality of their review, to be informed of the editorial decision on the reviewed manuscript, and to be given access to the comments of the other reviewers of the manuscript in question. In the survey conducted by Springer in 2014, reviewers stressed the importance of modesty in rewards, suggesting that reward should rely on the quality instead of the quantity of reviews and that stronger recognition of their work could motivate them to accept more invitations to review. ${ }^{7}$

\section{Incentives}

Although the impact of financial incentives on the quality of review is difficult to ascertain, one could expect an improved quality since a reviewer would probably spend more time on a review if he/she was remunerated for this activity. However, financial incentives would not 
be effective for reviewers overwhelmed with work. ${ }^{5}$ The expected improvement in quality has also been questioned. Squazzoni et al, conducting an innovative experiment to model the peer review process, concluded that offering material rewards to reviewers tends to decrease the quality and efficiency of the reviewing process. $^{8}$ Moreover, a reviewer may accept reviews in areas in which he/she is not very knowledgeable because of the financial motivation.

Other solutions have been suggested, such as free access to the journal content for subscription-based journals, discount for upcoming submissions for open-access journals, or free colour figures for future submissions. ${ }^{9,10}$ However, these solutions only work if the reviewer does not already have access to the content of the journal, which is becoming rare in developed countries due to standardized full access through institutional subscriptions. These solutions remain valuable in low-income countries.

\section{Recognition metrics}

Several recognition metrics have been designed since the emergence of problems with the peer review process. The first recognition platform was launched by Simon Gosling, the 2012 winner of the Peer Review Challenge organized by Elsevier. ${ }^{11}$ He suggested introduction of a standardized way to recognize the cumulative effort of a particular reviewer with a scheme based on badges and rewards, to be called the Elsevier Reviewer Badges and Rewards system. ${ }^{12}$ With this system, Elsevier delivers certificates to registered reviewers, which could particularly help researchers early in their career. Other similar initiatives have been launched: in April 2014, ORCID initiated a community working group to find solutions for rewarding reviewers. Following the suggestions of this group, ORCID launched a new peer review function in 2015 to recognize various peer review activities (review of publications, conference abstracts, and grants). Upon permission from the reviewer, organizations can attach reviewing information to this reviewer's ORCID record, which can then be shared with other organizations. With this function, ORCID hopes to improve scholars' recognition for their peer review work, so that they can possibly use it during their career.

Another platform called Publons was launched in 2014 aiming to turn peer review into a measurable research output by giving publishers the opportunity to track, verify, and showcase their peer reviewers' contributions. ${ }^{13}$

To overcome the long review process, eLife, an online open-access journal launched in 2012, is relying on internal reviewers from the editorial board, a system that in the end goes against the gold standard based on external solicitations. ${ }^{14}$

To face all the issues of the peer review process, several solutions have been suggested in the last few years. Are they enough to overcome all the challenges? How is the peer review process considered by different generations nowadays?

\section{A three-generation viewpoint}

\section{Emeritus Professor in Hepatogastroenterology (born in 1943)}

"As an old timer medical investigator, I went through the classical steps of an academic career, which included evaluation of peers and trainees. Throughout this time I lived under the dictat of peer review: "publish (well) or perish". I entered research early and luckily at the very start of a boom in my research field, viral hepatitis, which was blessed by waves of major successes affecting worldwide public health (blood screening, hepatitis B virus (HBV) vaccine, hepatitis C virus (HCV) discovery and $\mathrm{HCV}$ cure, and soon $\mathrm{HBV}$ cure).

This provided me with the opportunity of joining prestigious teams, witnessing and sharing those milestones. All this convinced me that "research is competition" and its meter is peer review. This vivid awareness pushed me to keep in the race.

Lately, the digital revolution combined with the publication drive has increased exponentially the publication output. Now scientometric analyses are needed to reveal confounding factors of bibliometry, to keep track of this peer review meter.

Unfortunately, like in sports or in evaluation of medicines, the peer review system is flawed and therefore desperately in need of improvement via innovations. These cannot be reduced to honoraria but have to rely on due recognition and reward of the expertise and involvement of the panel of contributors/ reviewers. New tools are warranted to achieve this.

The alleged words of Churchill on democracy "the worst form of government except for all the others" may also apply to peer review."

\section{PhD in Epidemiology, Biostatistician and Medical Writer (born in 1963)}

"Within the scientific community, I now belong to the older generation, the one that was told to review for a "better science". As for many scientists, my experience in peer review started by doing somebody else's reviews. I initially hated this time-consuming task, especially since I could not see much benefit in doing it. One of the arguments advanced by my busy supervisor was that reviewing could give me the opportunity to learn something new on my topic of interest. As a result, I spent hours working for free and learning very little, with my name appearing nowhere in the process.

Fortunately, I then gradually received review solicitations of my own, which I really appreciated since I at last had the impression of becoming an expert! Moreover, this gave me the opportunity to decline my supervisor's review solicitations, arguing that I had my own reviews to perform and high workload. However, a busy calendar combined with an absence of review recognition and increased number of review offers became further barriers for systematically accepting to undertake reviews. The easiness of declining to review by a single click often helped me in my decisions.

Promotion and recognition of peer review are 
urgently needed, including for young ghost reviewers. Unfortunately, I am not as familiar with e-technologies as my kids and I am not (yet) addicted to social networks. However, while I am still spending much time trying to understand how these things work, I am very well aware that interactive networking is the key. Some metrics such as the h-index or the French SIGAPS score exist for measuring researchers' publication activity. Similar metrics for reviewing activity have been tested. Efforts should be pursued to generalize and make such scores easily and widely available".

\section{MD/PhD student, MSc Ecole Normale Supérieure (born in 1992)}

"As a young "kid" in the research community, I have reviewed several papers in recent years, from direct editor's solicitation but also from indirect solicitation, ie from my different busy research godfathers who believe that I am able to help them in this precious and honourable task. Peer review done by a co-worker instead of the "designated" reviewer is common in the research community and this process should be more recognized by acknowledging all involved researchers. I consider peer review of particular interest because of the scientific content, but also because it allows me to discover from other researchers new approaches to present results or new ways to address certain scientific issues. From my relatively short experience, this is a really precious task, but it already conflicts with other workload. On that point, as an active social networker and a supporter of the 3.0 revolution, I believe that developing a universal research social network, which would promote my engagement in the research community through reviewing, would motivate me more to accept to peer review. This process has for me a long journey ahead, only if we rapidly start a research social media revolution".

\section{Discussion}

Peer review has always been a part of the "daily routine" of researchers: as reported in the survey of Tite and Schroter, it is for them an opportunity to learn something new. ${ }^{5}$

However, the increased number of submitted manuscripts means that for a given researcher, it is not uncommon to receive several review invitations per week and for an editor to have to ask 10 or more colleagues to end up with two willing reviewers. ${ }^{15}$ This disequilibrium between the number of invitations and the reasonable time that a reviewer can spend on peer review needs to be addressed soon in order to sustain the peer review system.

A transition regarding reviewer recognition is hopefully ongoing. Working groups, yearly seminars such as the Peer Review Week, best reviewer awards, and recognition metrics with online social networks are contributing to finding solutions in their own way. Combining all or some of the above proposals would promote the peer review process in the community. A common research social network could connect journals and reviewers/authors, introduce awards, metrics and incentives, and simultaneously summarize for each researcher his or her own work such as publications, abstracts, and reviews. This approach could help to overcome the peer review problem by promoting at the same level the importance of publication and of peer review.

\section{Acknowledgements}

We would like to thank Philip Robinson for his helpful comments and editing advice.

\section{References}

1 Philosophical Transactions - the world's first science journal. The Royal Society Publishing. http://rstl.royalsocietypublishing.org (accessed 12 Jan 2018).

2 Farrell PR, Magida Farrell L, Farrell MK. Ancient texts to PubMed: a brief history of the peer-review process. Journal of Perinatology 2017;37:13-5. doi:10.1038/jp.2016.209

3 Baker M. 1,500 scientists lift the lid on reproducibility. Nature 2016;533:452-4. doi:10.1038/533452a

4 Peer Review Survey 2015: Key Findings. Publishing Research Consortium. http://publishingresearchconsortium.com/index. php/134-news-main-menu/prc-peer-review-survey-2015-keyfindings/172-peer-review-survey-2015-key-findings (accessed 12 Jan 2018).

5 Tite L, Schroter S. Why do peer reviewers decline to review? A survey. Journal of Epidemiol Community Health 2007;61:9-12. doi:10.1136/ jech.2006.049817

6 Warne V. Rewarding reviewers - sense or sensibility? A Wiley study explained. Learned Publishing 2016;29:41-50. doi:10.1002/leap.1002

7 Carver C. We surveyed 5,000+ reviewers in 2015 and discovered.... Springer Source. 2015.http://www.springersource.com/we-surveyed5000-reviewers-in-2015-and-discovered/ (accessed 12 Jan 2018).

8 Squazzoni F, Bravo G, Takács K. Does incentive provision increase the quality of peer review? An experimental study. Research Policy 2013;42:287-94. doi:10.1016/j.respol.2012.04.014

9 Gasparyan AY, Gerasimov AN, Voronov AA, et al. Rewarding peer reviewers - maintaining the integrity of science communication. Journal of Korean Medical Science 2015;30:360. doi:10.3346/ jkms.2015.30.4.360

10 Mahian O, Wongwises S. How to encourage best peer reviewers: an empiric approach. Journal of Korean Medical Science 2015;30:506. doi:10.3346/jkms.2015.30.4.506

11 Lehane C. Peer Review Challenge - Winners unveiled. Elsevier Editors Update. 2012.https://www.elsevier.com/editors-update/story/peerreview/peer-review-challenge-winners-unveiled (accessed 12 Jan 2018).

12 van Dijk U. Reviewer Badges: Claim your badge and use it to highlight the journals for which you have reviewed. 2013.https://www.elsevier. com/reviewers-update/story/peer-review/reviewer-badges (accessed 12 Jan 2018).

13 Van Noorden R. The scientists who get credit for peer review. Nature Published Online First: 9 October 2014. doi:10.1038/ nature.2014.16102

14 Schekman R, Patterson M, Watt F, et al. Launching eLife, Part 1. eLife 2012;1. doi:10.7554/eLife.00270

15 Heinemann L. Reviewer: an endangered species?! Journal of Diabetes Science and Technology 2015;9:167-8. doi:10.1177/1932296814563883 